\title{
Search for effective models of stripes in the cuprates
}

\author{
Oleg Tchernyshyov and Leonid P. Pryadko ${ }^{\text {a* }}$ \\ anstitute for Advanced Study, School of Natural Sciences, \\ Olden Lane, Princeton, NJ 08540, USA.
}

\begin{abstract}
We argue that effective 1D models of stripes in the cuprate superconductors can be constructed by studying ground states and elementary excitations of domain walls in 2D model antiferromagnets. This method, applied to the $t-J$ model with Ising anisotropy, yields two such limiting cases: an ordinary $1 \mathrm{D}$ electron gas and a 1D gas of holons strongly coupled to transversal fluctuations of the stripe.
\end{abstract}

Formation of charge stripes in some of the cuprate superconductors [1] is a peculiar phenomenon in its own right. A possible connection between the stripes and high-temperature superconductivity makes them even more attractive to a theorist. The puzzle is nevertheless quite hard: to date there is no microscopic theory describing the physics of stripes in the cuprates.

Some progress has been made. Hartree-Fock studies of the Hubbard model near half-filling [2] have revealed that doped charges segregate in the form of narrow stripes. The stripes are domain walls separating antiferromagnetic (AF) domains with opposite orientations of the Neel vector, in agreement with experiments. However, the predicted linear density of charge on a stripe, $\nu=1$ hole per unit cell, indicates that there are no charges able to carry current. Experimentally, $\nu \approx 1 / 2$ for non-overlapping stripes [3].

In the absence of a microscopic theory, it is reasonable to look for a model description in which a stripe is a $1 \mathrm{D}$ object interacting with the surrounding antiferromagnet. A stripe is characterized by a ground state, by its elementary excitations, and by the interactions of the excitations among themselves and with the environment.

\section{The strategy}

A stripe is a complicated object. Its degrees of freedom may include charge and spin of the stripe particles, as well as transverse fluctuations of its

\footnotetext{
*Research supported in part by DOE Grant DE-FG0290ER40542.
}

position.

One potentially promising route to finding the right 1D model is to study simple solvable models of an antiferromagnet in 2D in the presence of a domain wall. A single domain wall can be created artificially, e.g., by wrapping a system with an odd number of rows on a cylinder. One may then study an isolated stripe at any filling $\nu$ by adding or removing the right number of electrons.

A 2D model may look unrealistic, but with universality and a bit of luck the resulting $1 \mathrm{D}$ theory may have just the right symmetry of the vacuum and the correct quantum numbers of elementary excitations.

\section{An example}

Consider a $2 \mathrm{D}$ model, a variant of the $t-J$ model in the Ising limit $J_{z} \gg J_{\perp}, t$ 朋:

$$
\begin{aligned}
H & =\sum_{\left\langle\mathbf{r} \mathbf{r}^{\prime}\right\rangle}\left[-t a_{\sigma}^{\dagger}\left(\mathbf{r}^{\prime}\right) a_{\sigma}(\mathbf{r})+\frac{J_{\perp}}{2} s_{+}\left(\mathbf{r}^{\prime}\right) s_{-}(\mathbf{r})\right. \\
& \left.+ \text { H. c. }+J_{z} s_{z}\left(\mathbf{r}^{\prime}\right) s_{z}(\mathbf{r})+V n\left(\mathbf{r}^{\prime}\right) n(\mathbf{r})\right]
\end{aligned}
$$

with the usual exclusion of doubly occupied sites. The dominating $J_{z}$ term minimizes the number of frustrated (ferromagnetic) bonds and thus sets the ground state. The energy of an AF bond $V-J_{z} / 4$ controls interactions of charged quasiparticles on the domain wall and in the bulk. Making $V-J_{z} / 4 \gg t>0$ prevents phase separation in the bulk, as well as on a stripe.

The physics of a stripe in this model is very different in the limits $\nu \rightarrow 1$ and $\nu \rightarrow 0$. 


\section{1. $\nu \rightarrow 1$ : $1 \mathrm{D}$ electron gas}

Assuming a domain wall of length $L$ has been created by frustrating boundary conditions, we first remove $L$ electrons. The holes end up on the wall forming a zigzag stripe with $\nu=1$.

For $\nu=1-\varepsilon$, the stripe will contain $\varepsilon L \ll L$ quasiparticles with the quantum numbers of electrons, $Q=-1, S_{3}= \pm 1 / 2$ (Fig. 11). Because of the zigzag geometry of the stripe, the electron quasiparticles feel a strong (of order $J_{z}$ ) staggered magnetic field. It confines electrons with opposite spins to different rows of the stripe. Within its row, an electron can delocalize to reduce kinetic energy. In the tight-binding model (11), such hopping is a multistage process, which involves pushing a neighbor spin out of the way.

Despite a suppressed hopping amplitude, the system can be described a dilute 1D electron gas.
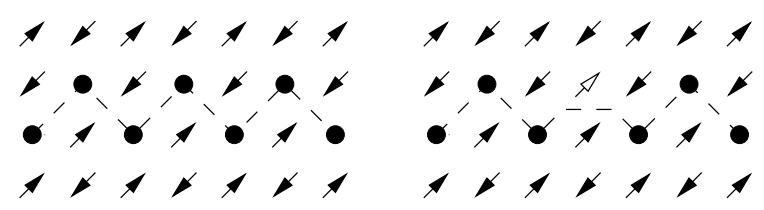

Figure 1. A zigzag stripe in the $t-J_{z}$ model. Left: $\nu=1$. Right: $\nu=1-\varepsilon$. Solid circles are sites with electrons removed. The dashed line is the location of the domain wall. The open arrow denotes an electron quasiparticle.

\section{2. $\nu \rightarrow 0$ : 1D holon gas}

Precisely at half-filling $(\nu=0)$, the domain wall is bond-centered. A single doped hole ends up at the domain wall (Fig. 2, left). If it starts to move alng the wall, two remarkable things happen. First, the hole leaves its spin $S_{3}= \pm 1 / 2$ behind in the form of a spinon and then propagates freely as a spinless object (a holon, Fig. 2, right). No additional frustrated bonds are produced afterwards. Furthermore, no costly spinons would be left behind had we started with 2 holes. Second, the holon (as well as the spinon) resides on a transverse kink of the domain wall. These elementary excitations are thus maximally strongly coupled to transverse fluctuations of the stripe. Kink direction can be specified by assigning a holon (or a spinon) a transverse flavor $\pm 1 / 2$ [5].

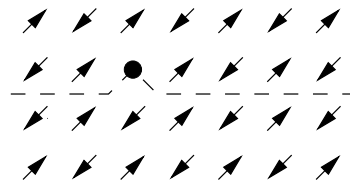

hole

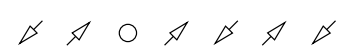

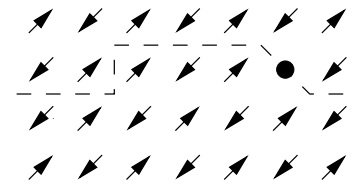

spinon

holon $\Delta \triangleleft \triangleleft \nabla \triangleleft 0 \nabla$
Figure 2. A bond-centered stripe near $\nu=0$ in the $t-J_{z}$ model. A doped hole (left) splits into a spinon and a mobile holon (right). Open symbols at the bottom denote spin and charge integrated across the domain wall with a smooth envelope function.

Holons are, in fact, the elementary excitations of the model, at least at low doping $\nu \ll 1$ : their kinetic energy is lower than that of immobile holes.

Finally, we note that holons with transverse flavor have also been found at small $\nu$ in a HartreeFock study of the Hubbard model [6].

\section{REFERENCES}

1. J. M. Tranquada, J. Phys. Chem. Solids 59, 2150 (1998).

2. See, e.g., H. J. Schulz, J. Phys. (Paris) 50, 2833 (1989).

3. K. Yamada et al., Phys. Rev. B57, 6165 (1998).

4. S. A. Kivelson, V. J. Emery, and H. Q. Lin, Phys. Rev. B42, 6523 (1990).

5. J. Zaanen, O. Y. Osman, and W. van Saarloos, Phys. Rev. B58, R11868 (1998).

6. O. Tchernyshyov and L. P. Pryadko, condmat/9907472 (unpublished). 